\section{Graphene jiggles up and down}

Nanometre-scale

measurements have revealed, with record precision, how atom-thick layers of carbon called graphene vibrate vertically.

Paul Thibado of the University of Arkansas in Fayetteville and his colleagues used a scanning tunnelling microscope to study how a free-standing sheet of graphene moves owing to random thermal fluctuations, on the scale of a single atom. They found that over nearly 3 hours, the membrane continually vibrates by as much as 10 nanometres, interposed with occasional larger flips as the sheet inverts its curvature. The team also used current from the microscope tip to control and enhance the motion.

Such vibrations could one day be exploited on a large scale in applications such as nanoscale power generation. Phys. Rev. Lett. 117, 126801 (2016)

\section{AGRICULTURE}

\section{Maize engineered to kill pest}

A bacterial protein protects maize (corn) from a major insect pest that has grown resistant to other insecticides.

The larvae of western corn rootworm feed on maize roots, and cause substantial crop losses across North America and Europe. Transgenic 'Bt' maize plants expressing insecticidal proteins from the soil bacterium Bacillus thuringiensis can fend off the

pest, but some worms have become resistant to certain

Bt proteins. Lu Liu at DuPont Pioneer in Hayward, California, and his colleagues analysed soil samples and identified a small protein made by Pseudomonas chlororaphis that killed rootworm larvae but not other common insects. Maize plants engineered to make this protein suffered little damage from the rootworm, regardless of whether or not the pest was sensitive to Bt proteins. Science http://doi.org/bqzh (2016)

\section{Early star-forming gas found}

Astronomers have identified distant gas-rich galaxies that probably caused the Universe's rate of star formation to peak some 10 billion years ago.

Several teams used the Atacama Large Millimeter/ submillimeter Array (ALMA) telescope in Chile to probe a well-studied patch of sky called the 'Hubble Ultra Deep Field', which offers the deepest view into the early Universe. Roberto Decarli at the Max Planck Institute for Astronomy in Heidelberg, Germany, and his colleagues found galaxies rich in carbon monoxide gas, which fuels star formation. The farther back in space and time the scientists looked, the more gas there was - corresponding to the burst of new stars that began about 3 billion years after the Big Bang.

The work is the first to reveal these long-expected, but never before seen, clumps of cool gas in the early Universe. Astrophys. J. in the press. Preprints at http://arxiv.org/ abs/1607.06770; http://arxiv. org/abs/1607.06771 (2016)

\section{Ants get addicted to morphine}

Ants get hooked on morphine much like humans do, showing similar changes in behaviour and brain chemistry.

Until now, only mammals have been shown to seek out addictive drugs in the absence of a

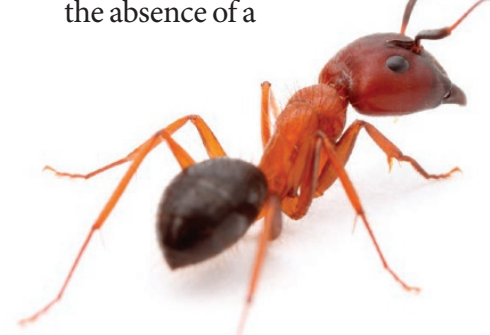

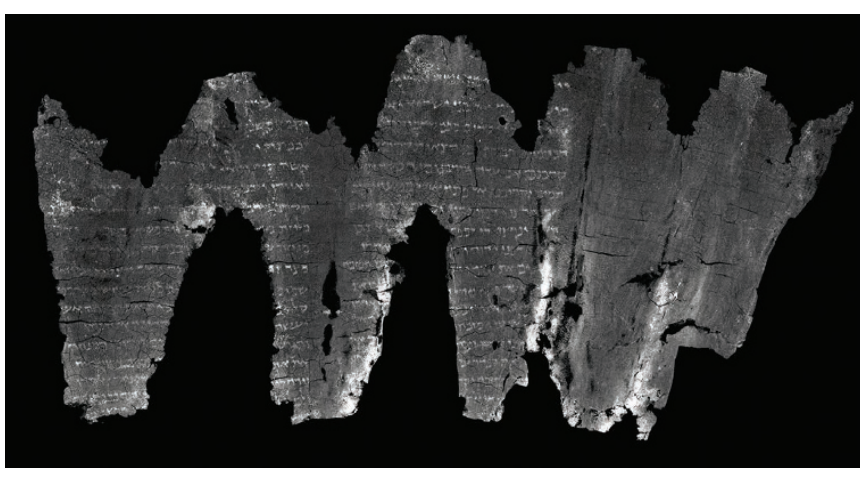

concurrent natural reward such as sugar. Brian Entler, Timothy Cannon and Marc Seid at the University of Scranton in Pennsylvania provided the ant Camponotus floridanus (pictured) with sugar water laced with the drug, then, over the course of several days, reduced the sugar concentration to zero while increasing the morphine level. The team then gave the ants a choice between sugar water and a sugar-free morphine solution. Of the ants exposed to morphine, $65 \%$ preferred the drug - roughly twice the rate seen in unexposed animals. The brains of morphinetrained ants also showed elevated levels of dopamine, a neurotransmitter associated with reward.

The authors suggest that ants could be used as a model to study addiction in humans. J. Exp. Biol. 219, 2865-2869 (2016)

\section{COMPUTER SCIENCE}

\section{Ancient scroll virtually unrolled}

Researchers have revealed the hidden contents of a fragile and damaged biblical scroll, thanks to computer scanning and imaging techniques.

The En-Gedi scroll dates back to at least the fourth century $\mathrm{AD}$ - the oldest Hebrew scroll found, other than the Dead Sea Scrolls - and cannot be unrolled without causing further damage. William Seales at the University of Kentucky in Lexington and his colleagues used X-rays to scan the scroll, and developed software, called "virtual unwrapping", to digitally reconstruct the 'pages' and words of the ancient artefact (pictured). They found that the ink-based text, discovered in 1970, contains fragments of the Book of Leviticus. Sci. Adv. 2, e1601247 (2016)

\section{ECOLOGI}

\section{Rats and cats drive extinctions}

Non-native predatory mammals such as cats and rats can wreak havoc on native animal populations, especially on islands.

Tim Doherty of Deakin University in Melbourne, Australia, and his colleagues studied the International Union for Conservation of Nature (IUCN) Red List of threatened species. They found that $58 \%$ of extinctions of birds, mammals and reptiles recorded in the past 500 years can be blamed, at least in part, on exotic mammalian predators, including dogs and pigs. In particular, rodents helped to drive 75 species extinct, and cats 63. Moreover, almost 600 species, most of them island-dwellers, are currently threatened to some degree by non-native mammalian predators.

The authors stress the importance of efforts to eradicate exotic predators from islands around the world. Proc. Natl Acad. Sci. USA http:// doi.org/bqwb (2016)

\section{$\rightarrow$ NATURE.COM}

For the latest research published by Naturevisit:

www.nature.com/latestresearch 